\title{
A course on foundations of optical system analysis and design (FOSAD)
}

\section{Lakshminarayanan Hazra}

Lakshminarayanan Hazra, "A course on foundations of optical system analysis and design (FOSAD)," Proc. SPIE 9289, 12th Education and Training in Optics and Photonics Conference, 92890N (17 July 2014); doi: $10.1117 / 12.2070381$

SPIE Event: 12th Education and Training in Optics and Photonics Conference, 2013, Porto, Portugal 


\title{
A Course on Foundations of Optical System Analysis and Design (FOSAD)
}

\author{
Lakshminarayan Hazra \\ Department of Applied Optics and Photonics \\ University of Calcutta \\ 92 Acharya Prafulla Chandra Road, Kolkata 700 009, India
}

\begin{abstract}
Optical system analysis and design constitute one of the core activities in optical engineering. This activity is currently carried out with readily available software. Notwithstanding the significant roles played by the latter in bringing about a paradigm shift in the field, proper appreciation and efficient use of software call for knowledge and understanding of the physical principles involved in optical system analysis and design. A large number of excellent books and publications by experts deal with different aspects of the problem. However, newcomers in the field, and practicing analysts and designers with no formal training in the subject feel bewildered by the plethora of information. The course on 'Foundations of Optical System Analysis and Design' is contemplated to alleviate the problem.
\end{abstract}

Keywords: Lens Design, Optical System Analysis, Optical System Design, Teaching of Optics

\section{INTRODUCTION}

Ready availability of powerful software has brought about a metamorphosis of the field of optical system analysis and design, one of the core areas of optical engineering. Practicing scientists or technologists are no longer constrained by their limited computational ability; rather they can give vent to their imagination and carry out experiments with intensive numerical procedures in pursuance of practical problems in analysis and synthesis of complex optical and photonic systems. Indeed, someone with good understanding of the principles of image formation and image quality assessment procedures, photometry, radiometry, basics of aberration theory and characteristics of different types of lens systems, optical and photonic components, sources and detectors can do wonders with many of the software.

Unfortunately, many scientific and/or technical people or their managers seem to have a short sighted perception that procuring powerful optics design software is sufficient for tackling practical problems of analysis and synthesis of optical systems with rudimentary knowledge of optics. This notion has gained ground, because even a novice often comes out with makeshift solutions for routine or known problems by using the software. Unless proper attention is given for assessment of quality of the solution so obtained, the suboptimal nature of the makeshift solution will be realized neither by the inexperienced designer, nor by the unwary user of the design. This is one of the major drawbacks of self-learning mode for optical design, particularly so in the beginning. On the other hand, there is a burgeoning requirement for novel optical and photonic systems catering to needs of diverse fields from defense, aerospace, astronomy and health sciences, to name a few. Emulating routine systems is often inadequate to tackle such problems.

Gone are the days when only suitably trained professionals used to carry out optical system analysis and design. Very few centers of learning are now conducting degree level courses that impart lessons on optical system analysis and design. The shortfall in the supply of trained designers is being increasingly met up by physicists and engineers from diverse backgrounds. The latter are being compelled to take up the unenviable task with little support from their colleagues and seniors. They are on the lookout for sources that can provide answers to their queries.

On a different note, scientists working in different frontline areas are increasingly making use of optics and photonics in pursuance of their objectives, and often in the process they have to carry out novel experimental setups. It is obvious that proper conceptualization and correct implementation of the experiments call for understanding of characteristics and behavior of the optical and photonic components and devices to be used and of the optical system being developed and used in course of the experiment. Lack of this understanding is often a big hurdle.

12th Education and Training in Optics and Photonics Conference, edited by

Manuel F. P. C. Martins Costa, Mourad Zghal, Proc. of SPIE Vol. 9289, 92890N

(C) 2014 SPIE, OSA, IEEE, ICO · doi: 10.1117/12.2070381

Proc. of SPIE Vol. $928992890 \mathrm{~N}-1$ 
Some of the problems mentioned above have been highlighted recently with suggestions for remedial actions ${ }^{1-6}$. SPIE, OSA and few other societies are also providing short duration tutorials by experts during their conferences as continuing education programs.

With this backdrop in view, a set of ten lecture units or modules dealing with foundations of optical system analysis and design (FOSAD) has been developed. Primary emphasis is given for treatment of imaging optical systems. In a regular optical engineering course, this is covered in two semesters with accompanying tutorials. For special purposes, the whole course can be squeezed into twenty lecture hours, with two lecture hours assigned for each of the ten modules, accompanied by tutorials. This course has so far been delivered in Poland, Japan and India, and it is being modified by feedbacks from participants.

\section{GOALS OF FOSAD}

The goals of the course on FOSAD are:

(i) To impart basic knowledge of ray and wave optics for understanding behavior of optical systems

(ii) To emphasize the varying levels of approximation, and their needs for handling the seemingly intractable (in rigorous mathematical sense) problem of optical system design

(iii) To underscore the role of various types of symmetry in (a) axial imaging, (b) extra-axial imaging, (c) development of aberration theory, and (d) structure of lenses

(iv) To reiterate the role of pupils in analysis and synthesis of optical systems

(v) To dispel commonly prevalent myths and misconceptions in instrumental optics

(vi) To demystify 'Automatic Lens Optimization'

(vii) To reduce the role of empiricism and heuristics in practical lens design

(viii) To provide adequate references for seeking answers to queries not properly addressed in the course

(ix) To bridge the gap between 'examination optics' and 'real optics' [- a goal set forth by late A. E. Conrady ${ }^{7}$ about a century back]

(x) To highlight the great contributions of pioneers and stalwarts in the field [many of these contributions are currently getting into oblivion, but are likely to infuse lateral thinking in curious onlookers]

\section{STRUCTURE OF THE MODULES OF FOSAD}

\subsection{Module I General Introduction}

Different types of optical systems used in astronomy, biology, industry, defense, entertainment etc.

Optical and photonic components and devices used in the systems

Specifications for systems, components and devices

Demonstration of different lens types

Ray layouts for representative systems upholding the unity of functions in the apparent diversity

ICO definition of "Optics"

Theories of Light used in analytical treatment of optical systems

Interrelationship between the theories; What? Where? When?

Characteristics of a propagating plane light wave: polarization, amplitude, direction, frequency and phase

Concept of Wavefront and Rays as orthogonal trajectories

Statement of the problem of lens design/optical system design

General Methodology for Optical Design

\subsection{Module II Paraxial Treatment}

Raison d'être of paraxial analysis 
Paraxial Approximation: on-axis imaging and extra-axial imaging

Paraxial Ray Tracing Formulae

Choice of paraxial ray variables

General Object-Image relationship for an axi-symmetric imaging system

Finite focal and afocal systems

Principal planes and Nodal planes; concept of equivalent focal length

Conjugate positions for afocal systems; types of afocal systems

Throw-Magnification Curve

Telephoto and Wide angle Lenses

\subsection{Module III Further Topics in Paraxial Optics}

Importance of paraxial invariant

Stops and Pupils

Vignetting

Telecentric systems

Definition of F-number and numerical aperture

Role of Pupil Matching for Optical systems in tandem

Field Lens and Relay Lens

Medical Endoscopes: A Case study

Classification of lens systems based on field size and relative aperture size

Types of telescopes

Magnification by simple magnifiers; magnifiers and eyepiece

Compound Microscopes; Infinity corrected objectives

Illumination Systems: Netsonian critical illumination and Köhler illumination

Eyepieces; optical parameters of the human eye

Monocular, Binocular and Biocular Viewing

\subsection{Module IV Foundations of Aberration Theory}

Fermat's principle and Malus-Dupin Theorem

Introduction to Hamiltonian Optics

Optical Path Differential Theorem

Hamilton-Bruns Point Eikonal; Point Angle Eikonal; Angle Eikonal

Perfect Imaging with Real rays

Stigmatic Imaging of a point by Cartesian ovals

Aplanatic Surfaces

Optical Cosine Rule; Abbe sine condition; Herschel condition

Optics around a principal ray

Parabasal Optics; $s$ and t rays

Lagrangian Optics

\subsection{Module V Monochromatic and Chromatic Aberrations}

Image Formation by real rays in a general optical system without symmetry

Image Formation by real rays in an axi-symmetric lens system

Symmetry considerations in performance analysis: Axial symmetry and Bilateral symmetry

Measures of Aberrations

Ray Aberration, Wave Aberration and Hamilton-Bruns' eikonal : Interrelationships

Effects of shift of center and radius of the reference sphere

Power series expansion of the aberration function

Longitudinal Shift of focus and Transverse Shift of focus

Aberrations of Various orders

Point imaging Aberrations and Aberrations of image shape

Classification of Aberrations 
Chromatic Aberrations

Axial Chromatism and Lateral Chromatism

Secondary Spectrum

Conrady (D-d) formula

\subsection{Module VI Primary Aberrations}

Physical significance of primary aberrations

Primary Aberrations for a specific field point

Primary Aberrations and Seidel Aberrations

Seidel Aberrations in terms of paraxial parameters of the paraxial marginal ray and the paraxial pupil ray

(Expressions only; No derivation)

Summation of primary aberrations for cascaded interfaces

Ray diagrams corresponding to different primary aberrations

Aberration free lenses and surfaces

Thin Lens Aberration Theory

Seidel Aberrations of a thin lens in air with stop on it

Dependence on Shape and Conjugate variables

Dependence on refractive index and dispersion of the optical material of the lens

Stop Shift formulae

Pupil Aberrations

Conjugate Shift formulae

\subsection{Module VII Diffraction Theory of Image Formation}

Raison d'être for Diffraction Theory

Airy Pattern

Two point resolution; Rayleigh criterion; Sparrow Criterion

Point Spread Function (PSF) for Aberrated systems

Rayleigh quarter wavelength rule

Strehl criterion (Definitionshelligkeit)

Special case for small aberrations

Variance of Wave Aberration

Maréchal criterion for aberration balancing

Strehl criterion for large aberrations

Zernike Aberration polynomials

\subsection{Module VIII System Theoretic Viewpoints in Optical Image Formation}

Principle of Superposition

Space invariance and isoplanatism

Fourier Analysis

Physical interpretation of the kernel of Fourier Transform

Optical Transfer Function (OTF): Modulation Transfer Function (MTF) and Phase Transfer Function (PTF)

Edge Spread Function (ESF)

Interrelationship between PSF, OTF and ESF

Coherence and Incoherence

Abbe Theory of coherent image formation

Imaging as a process of double diffraction

Hopkins' aberration tolerance criterion based on OTF

Through focus MTF

\subsection{Module IX Lens Design Optimization}

Mathematical preliminaries for optimization of univariate and multivariate functions

Degrees of freedom in lens design optimization 
Boundary constraints based on physical realizability, manufacturability and material availability

Concept of Merit Function as a metric during optimization process

Nonlinear Optimization methods

Formation of Merit Function in terms of aberrations and pseudo-aberrations

Method of least squares optimization

Damped least squares and techniques for damping

Local optimization and Global optimization

Heuristic methods for global/quasi-global optimization

Stochastic global optimization methods

A prophylactic approach for global synthesis

\subsection{Module X Miscellaneous Topics}

(a) Conventional lens types for infinite conjugate systems

(b) Aberration Reduction strategies in lens design

(c) Advanced Topics in Extra-axial imagery: Paraxial and Real pupils; Pupil exploration; Anamorphic imagery and its effects on OTF analysis; Effects of residual aberrations on Cosine-fourth power law of illumination

(d) Advanced topics in Chromatism: Spherochromatism; Effects of Pupil chromatism; Image space associated rays and Object space associated rays; Polychromatic PSF and MTF

(e) Elements of Zoom Lenses

\section{CONCLUDING REMARKS}

The course on FOSAD presupposes that the audience has A-Level knowledge of optics, three dimensional geometry and multivariate calculus; an exposure to Fourier analysis is an added advantage. Also the course is supposed to play a complementary role in making effective use of any worthwhile optical analysis and design software to which the participant has access. Topics like ray tracing, derivation of Seidel aberrations and computation of finite ray aberrations are not covered, for these details do not enhance understanding the behavior of optical and photonic systems. A set of questions accompany each module; these are covered in the tutorials. Feedback is sought from each participant for tailoring of course content and orientation.

\section{REFERENCES}

[1] Shannon, R. R., “Teaching lens design”, Opt. Eng. 32(8), 1722-1724 (1993)

[2] Kidger, M.J., "The Importance of Aberration Theory in Understanding Lens Design," Proc. SPIE 3190, 26-32 (1997).

[3] O'Shea, D. C., “A survey of Lens Design Courses”, Proc. SPIE 3190, 75-81 (1997).

[4] Sasian, J. M., "Trends in Teaching Lens Design", Proc. SPIE 4588, 56-58 (2002).

[5] Bentley, J. L., "Thirty different views of a lens design solution space: a good example for teaching students how to design and not to design a lens", Proc. SPIE 5875, 58750C-1-58750C-8 (2005).

[6] Doushkina, V. V. and Silberman, D. M., Proc. SPIE 6668, 66680E-1 - 66680E-13 (2007).

[7] Conrady, A.E., [Applied Optics and Optical Design, Part I], Oxford, London (1929) [Reprinted by Dover, New York (1957)]. 